ISSN 0103-5150

Fisioter. Mov., Curitiba, v. 24, n. 4, p. 745-753, out./dez. 2011

Licenciado sob uma Licença Creative Commons

\title{
0 relacionamento interpessoal e a adesão na fisioterapia
}

\author{
The interpersonal relationships and physical therapy adherence
}

\section{Marina Medici Loureiro Subtil ${ }^{[\mathrm{a}]}$, Dominiqui Costa Goes ${ }^{[\mathrm{b}]}$, Tiago Cardoso Gomes ${ }^{[\mathrm{c}]}$, Mariane Lima de Souza ${ }^{[\mathrm{d}]}$}

[a] Fisioterapeuta, Mestre em Psicologia pela Universidade Federal do Espírito Santo (UFES), docente do curso de Licenciatura em Música da Universidade Federal do Espírito Santo (UFES), Vitória, ES - Brasil, e-mail: marina.fisio@hotmail.com

[b] Graduanda em Psicologia pela Universidade Federal do Espírito Santo (UFES), Vitória, ES - Brasil.

[c] Graduando em Psicologia pela Universidade Federal do Espírito Santo (UFES), Vitória, ES - Brasil.

[d] Psicóloga, Doutora em Psicologia pela Universidade Federal do Rio Grande do Sul, docente do Departamento de Psicologia Social e do Desenvolvimento e do Programa de Pós-Graduação em Psicologia da Universidade Federal do Espírito Santo (UFES), Vitória, ES - Brasil, e-mail: limadesouza@gmail.com

\section{Resumo}

Introdução: A relação estabelecida entre fisioterapeuta e paciente é um aspecto fundamental do processo de adesão no tratamento fisioterapêutico e neste estudo foi definida como um processo multifatorial. objetivos: Investigar a relação entre o fenômeno da adesão na fisioterapia e as características do relacionamento estabelecido entre fisioterapeutas e pacientes. Metodologia: Estudo qualitativo e exploratório, com delineamento ex-post facto, amostra por conveniência de 11 pacientes de 25 a 73 anos com diagnósticos clínicos variados; e quatro fisioterapeutas atuantes na equipe em que esses pacientes foram atendidos. Os participantes responderam a uma entrevista semiestruturada contendo tópicos sobre o processo de adesão na fisioterapia e o papel do relacionamento fisioterapeuta-paciente. A análise qualitativa dos dados seguiu os critérios da fenomenologia-semiótica. Resultados e discussão: Os relatos dos pacientes sugeriram um contexto temático para o fenômeno da adesão focado em duas áreas: a) caracterização da fisioterapia e do bom profissional e b) contraposição entre continuidade e interrupção do tratamento. Os relatos dos fisioterapeutas indicaram um contexto temático focado em três áreas: a) capacidades e habilidades do bom 
fisioterapeuta; b) adesão à fisioterapia; e c) relacionamento fisioterapeuta-paciente. A redução fenomenológica indicou três focos problemáticos para o fenômeno da adesão ao tratamento na fisioterapia: a necessidade do cuidado integral; a dificuldade na comunicação paciente-terapeuta; e as contingências socioeconômicas do paciente. A interpretação fenomenológica reforçou e relacionou os aspectos encontrados na redução. Conclusão: 0 processo de adesão na fisioterapia pode ser caracterizado como multifatorial e o relacionamento interpessoal, entre fisioterapeuta e paciente, como fator essencial ao sucesso da reabilitação e consequente adesão.

Palavras-chave: Adesão ao tratamento. Comunicação. Relacionamento. Fisioterapia. Reabilitação.

\section{Abstract}

Introduction: The study of the relations between therapists and patients contributes to the understanding of the issues involved in adherence phenomenon to physiotherapy. Objectives: To investigate the relation between the phenomenon of adherence in physiotherapy and the characteristics of the established relationship between therapists and patients. Methodology: Of qualitative and exploratory nature, based on the semioticphenomenological criteria of analysis. Took part in this study 11 patients, from 25 to 73 years old with varied clinical diagnoses, in addition to four physiotherapists active in a team that treated these patients. The participants answered a semi-structured interview containing topics about the adhesion process in physiotherapy and the role of therapist-patient relationship. Results and discussion: Data analysis followed the three steps of semiotic phenomenology: description, reduction and interpretation. The patient reports have suggested an organized structure of the phenomenon in two areas: a) the physiotherapy and the good professional and b) treatment adherence and non-adherence. The physiotherapists' description indicated a structure organized in three thematic areas: a) skills and abilities of the good therapist, b) adherence to physiotherapy, c) the physiotherapist and patient relationship. The phenomenological reduction suggests that the phenomenon of adherence to treatment in physiotherapy is structured around three aspects: comprehensive care, communication, and the contingencies of socio economic conditions of each patient. Conclusion: The adhesion process in physical therapy was characterized as multifactorial, and the interpersonal relationship between therapist and patient perceived as essential to successful rehabilitation and subsequent adherence to the Physiotherapy.

Keywords: Treatment adherence. Communication. Relationship. Physiotherapy. Rehabilitation.

\section{Introdução}

Os serviços de saúde oferecidos à sociedade geralmente são atravessados pelo relacionamento entre os profissionais que prestam o atendimento e os pacientes que o recebem. As pesquisas sobre essas formas de relacionamento têm contemplado, de forma geral, o relacionamento interpessoal médicospacientes e enfermeiros-pacientes, com ênfase nas questões ligadas ao processo de comunicação estabelecido entre essas partes durante o processo de adoecimento e tratamento (1-4).

Compreender os aspectos envolvidos no adoecimento do paciente é fundamental para a efetiva promoção da saúde, pois o enfermo passa a ser considerado em sua integridade física, psíquica e social, e não somente de um ponto de vista biológico (5). Nesse contexto, a habilidade de escutar o paciente é destacada por Marinho (6) como importante na relação terapêutica estabelecida, além de favorecer a criação de um espaço em que o paciente tem a permissão de expressar-se; e ambos, dessa forma, têm a possibilidade de participar efetivamente do processo de cura. 0 paciente pode se sentir, então, aceito, compreendido, amado e sem culpa. Por outro lado, a avaliação parcial desse paciente representa a fragilização da relação terapêutica (7) quando desconsidera que a eficácia terapêutica depende de todos os atos que envolvem o encontro entre o paciente e o profissional (8).

No contexto da fisioterapia, uma parte dos escassos estudos sobre o relacionamento profissionalpaciente (6-13) sugere que um dos fatores necessários para a efetividade do processo de reabilitação é a qualidade da relação entre o paciente e o fisiote- 
rapeuta. Isto é, à medida que os pacientes se envolvem no tratamento fisioterapêutico, relações interpessoais marcadas pela afetividade se desenvolvem e podem afetar o curso e a qualidade do tratamento em questão (14).

Nesse panorama, o desenvolvimento do relacionamento entre fisioterapeuta e paciente apresentase como algo natural e muito provável de acontecer entre essas partes, visto que o tratamento em questão apresenta fatores favoráveis ao surgimento de um relacionamento interpessoal, tais como: longo período de convivência, estímulos táteis prolongados e comunicação verbal em boa parte do atendimento fisioterapêutico.

Dessa forma, o objetivo deste estudo foi investigar a relação entre o fenômeno da adesão na fisioterapia e as características do relacionamento estabelecido entre fisioterapeutas e pacientes. Considerando a escassez de trabalhos sobre o tema no campo da fisioterapia, o estudo teve caráter exploratório, focalizando pacientes e fisioterapeutas de um centro de reabilitação pública do Espírito Santo.

\section{Metodologia}

O estudo seguiu um enfoque qualitativo (15), caracterizado como descritivo de acordo com seus objetivos e com delineamento ex-post facto conforme seus procedimentos técnicos (16).

Participaram do estudo 11 pacientes adultos de 25 a 73 anos, com diagnósticos clínicos variados, como, por exemplo, fraturas ósseas, degenerações articulares e reabilitação de pós-operatórios ortopédicos, que estavam em atendimento há pelo menos 10 sessões no centro de reabilitação; além de quatro fisioterapeutas atuantes na equipe em que esses pacientes foram atendidos.

O estudo foi aprovado pelo Comitê de Pesquisa do Hospital Nossa Senhora da Glória, seguindo as diretrizes e normas do Conselho Nacional de Saúde para a elaboração de pesquisas com seres humanos (196/96).

Após serem informados sobre a pesquisa, aqueles que aceitaram participar assinaram o Termo de Consentimento Livre e Esclarecido. Tanto os pacientes quanto os fisioterapeutas responderam a uma entrevista com um roteiro semiestruturado, contendo três tópicos sobre o processo de adesão na fisioterapia e o papel do relacionamento fisioterapeuta-paciente:
1) descrição da relação estabelecida entre pacientes e fisioterapeutas;

2) as razões para aderir ou não à fisioterapia;

3) influência do relacionamento no processo de adesão e no sucesso da reabilitação.

A análise dos dados seguiu os critérios da fenomenologia-semiótica, que propõe três etapas sinérgicas de avaliação das entrevistas: descrição, redução e interpretação. A descrição consistiu em uma separação de partes de um todo e em uma demarcação de unidades de sentido do texto original. A redução apresenta-se como uma síntese, já que agrupa as partes separadas da descrição apontando os temas centrais do fenômeno, que serão analisados posteriormente na interpretação. Já a interpretação organiza o conteúdo das unidades reduzidas em um texto que integra todos os temas que compõem a experiência de cada indivíduo dentro do fenômeno estudado, comparando-os com as pesquisas realizadas acerca da temática escolhida (17-18). Portanto, no presente artigo a descrição e a redução serão apresentadas a seguir, na seção resultados, com a descrição dividida em dois blocos: primeiramente, a percepção dos pacientes e, logo em seguida, a percepção dos fisioterapeutas. A interpretação fenomenológica será apresentada na seção discussão.

\section{Resultados}

\section{A percepção dos pacientes}

Os relatos dos pacientes sugeriram um contexto temático para o fenômeno da adesão ao tratamento focado na relação estabelecida entre o paciente e o fisioterapeuta e sua importância para o sucesso da reabilitação. Divide-se em duas temáticas: a) caracterização da fisioterapia e do bom profissional e b) contraposição entre continuidade e interrupção do tratamento.

\section{A caracterização da fisioterapia \\ e do bom profissional}

0 bom fisioterapeuta alia a habilidade técnica à habilidade para o relacionamento interpessoal. 0 fisioterapeuta é o profissional capaz de aliviar ou suprimir os sintomas dolorosos de cada doença, tornando 
seus pacientes capazes de voltar às atividades de vida diária. Além da competência técnica, esse profissional precisa saber relacionar-se com o paciente, oferecendo atenção individualizada, avaliando e cuidando daquele que o procura de uma forma integrada, considerando tanto os aspectos físicos como emocionais. Para ser considerado bom, o relacionamento entre paciente e fisioterapeuta precisa envolver empatia, reciprocidade, confiança e afeto. Todas essas qualidades foram percebidas como determinantes do relacionamento e do sucesso do processo terapêutico: "tem que ter conhecimento, mas tem que conversar, ouvir, dar atenção..." (Paciente 07). "Não adianta ser muito bom na teoria se não sabe nem conversar com o paciente e dar a atenção que ele precisa aqui..." (Paciente 02).

0 relacionamento entre os pacientes e os fisioterapeutas da unidade de atendimento foi percebido como satisfatório, embora o tempo de contato com o profissional, bem como a atenção dispensada por ele tenha sido avaliada como aquém das expectativas do paciente: "eu sei que eles atendem muitos pacientes por hora, e acho que talvez por isso não possam nos dar a devida atenção, passar mais tempo nos escutando, avaliando e tudo mais" (Paciente 09).

\section{A contraposição entre continuidade e interrupção do tratamento}

A adesão ao tratamento é um processo vinculado tanto ao paciente quanto ao profissional. Realizar o tratamento fisioterapêutico até o final é uma tarefa que requer comprometimento tanto daquele que trata quanto daquele que é tratado. No caso dos próprios pacientes, fatores como: disciplina, desejo de melhorar, assiduidade, confiança no profissional e na técnica escolhida, compromisso e realização das orientações quanto à execução das atividades domiciliares figuram como determinantes para que o paciente realize todo o tratamento na fisioterapia. No caso dos fisioterapeutas, fatores relacionados à qualidade do relacionamento e às habilidades sociais do terapeuta aparecem como prioritários, inclusive em relação às habilidades técnicas. Oferecer atenção, carinho, respeito, amor, confiança e afeto foi visto pelos pacientes como ação essencial para sentirem-se motivados a continuar a fisioterapia.

$\mathrm{O}$ abandono da fisioterapia aparece ligado a quatro fatores: dificuldades financeiras; necessidade de voltar ao trabalho; falta de interesse e desvalorização do tratamento; insatisfação com as técnicas fisioterapêuticas e com o relacionamento com o fisioterapeuta. A falta de interesse e a desvalorização do tratamento decorrem tanto de uma avaliação negativa dos serviços oferecidos pelo SUS quanto de aspectos psicológicos do próprio paciente. Dentre os aspectos psicológicos, destaca-se a dificuldade em lidar com o processo de adoecimento e aceitar um corpo doente com incapacidades físicas, além de um estado de humor depressivo. A desvalorização do atendimento oferecido pelo SUS tem início em crenças fundamentadas na relação de que o que é gratuito e oferecido pelo SUS não teria qualidade. Esses aspectos contribuem para o descomprometimento do paciente com o tratamento oferecido.

\section{A percepção dos fisioterapeutas}

Os relatos dos fisioterapeutas sugeriram um contexto focado em três áreas temáticas: a) as capacidades e habilidades do bom fisioterapeuta; b) a adesão à fisioterapia; c) o relacionamento fisioterapeutapaciente.

\section{As capacidades e habilidades} do bom fisioterapeuta

As qualidades que o profissional deve ter para oferecer um tratamento satisfatório se referem tanto ao contexto técnico quanto ao contexto pessoal. O bom fisioterapeuta é aquele profissional capaz de associar suas capacidades e habilidades técnicas a capacidades e habilidades comunicativas, tais como: saber escutar e compreender o paciente em estado de sofrimento, oferecendo atenção e cuidado em forma de afeto (amor, respeito, solidariedade/apoio, carinho, atenção, escuta) e de contato físico (terapia manual). A atuação fisioterapêutica não deve permanecer restrita à execução de técnicas e à aplicação de aparelhos, mas deve construir uma abordagem que integre as competências profissionais e as habilidades para lidar com o outro de forma abrangente.

\footnotetext{
(...) ter respeito pelo paciente, porque ele precisa do seu toque e do seu carinho, da sua atenção, do seu conhecimento técnico científico. Mas não adianta nada saber todas as técnicas se você não encosta no paciente. A maioria dos nossos pacientes precisa mais da nossa
} 
atenção, do nosso calor humano, do que às vezes da nossa técnica, e só de você encostar e tocar nele, de conversar com ele, você já está resolvendo parte do problema (Fisioterapeuta 04).

O fisioterapeuta foi descrito como o profissional capaz de devolver as aptidões físicas ao paciente, possibilitando o retorno às suas funções de vida diária, ao trabalho e levando a uma melhora da qualidade de vida. 0 bom fisioterapeuta é aquele que atua desde a prevenção de disfunções até a reabilitação do indivíduo, orientando os pacientes para executar as atividades em casa, já que as duas horas semanais na fisioterapia são insuficientes para a reabilitação completa e rápida do paciente.

Ser um bom profissional remete à capacidade de aliviar a dor do outro, tendo como foco as dimensões relacional, afetiva, criativa e nobre atribuídas à profissão.

\section{A adesão à fisioterapia}

O processo de adesão na fisioterapia é um evento associado em grande parte ao paciente, considerando esse último peça fundamental no processo de reabilitação. 0 paciente deve ser um agente ativo desse processo, desejando alcançar e buscar a melhora e reabilitação das funções perdidas ou prejudicadas pelo adoecimento. Para que ele permaneça no tratamento, é necessário, por parte dos fisioterapeutas, tratar com atenção cada caso apresentado, de forma individualizada, oferecendo a assistência adequada àquele caso: para ele continuar a vir ele precisa se sentir apoiado, cuidado, ouvido, tratado (Fisioterapeuta 02).

A carência por afeto e os demais comprometimentos de ordem emocional dos pacientes foram apontados como fatores ligados ao processo de adesão.

Às vezes não são só problemas físicos, tem problemas de família desorganizada, com dores físicas e dores da alma. Alguns vêm à fisioterapia só para saírem de casa e se sentirem menos sozinhos e abandonados, eles fogem do ambiente em que vivem (Fisioterapeuta 01).

Os fatores de ordem emocional foram identificados como limitantes na continuidade do tratamento caso não sejam solucionados. Cabe ao fisioterapeuta identificar essas possíveis alterações e encaminhar ao profissional de psicologia da equipe.
0 processo de não aderir ao tratamento fisioterapêutico esteve associado a seis causas principais: às condições socioeconômicas desfavoráveis, dificultando o acesso ao local de tratamento; à busca pela aposentadoria precoce e o auxílio do INSS; à demora até o início do tratamento; à não aceitação do quadro crônico de certas doenças; à melhora relativa dos sinais e sintomas da doença somada à falta de persistência em continuar até o final; e à necessidade de voltar às atividades de trabalho para o sustento da família.

Portanto, o processo de aderir ou não à fisioterapia foi relacionado tanto a contingências gerais quanto a características pessoais ou psicológicas dos pacientes. Nesse processo, o paciente é visto como elemento principal e o fisioterapeuta como elemento secundário. Ser o elemento principal significa ter a maior parte das responsabilidades pelo processo de reabilitação, isto é, a melhora do quadro é resultado mais da vontade e do interesse do paciente em alcançar os objetivos do tratamento do que todo o tratamento em si oferecido pelos fisioterapeutas.

\section{O relacionamento fisioterapeuta-paciente}

0 relacionamento entre fisioterapeuta e paciente é um fator fundamental para o sucesso da reabilitação, além de ser peça-chave no processo de adesão. A relação que se constrói entre esses indivíduos deve ser permeada por respeito, carinho, empatia, atenção e capacidade de escuta do outro: a gente tem que ter empatia com o paciente, tem que dar atenção. Os pacientes vêm pra cá procurando o tratamento de reabilitação, mas a gente não é só fisioterapeuta, a gente acaba sendo um pouco de conselheiro (Fisioterapeuta 02).

A relação é descrita tanto como uma amizade com características profissionais, mas sem a formação de vínculo íntimo, quanto como uma relação com forte vínculo, mas sem necessariamente caracterizar uma relação de amizade. Isto é, o vínculo ora apresentase como amizade e ora como relacionamento profissional, associando características dos dois tipos. Independente de como é caracterizado, o relacionamento entre fisioterapeuta e paciente é percebido como fator essencial no processo de reabilitação e adesão na fisioterapia.

Eu converso, tento formar não uma amizade, mas um vínculo com o meu paciente, eu gosto de conversar, de 
ouvir o que ele tem a dizer. Não precisa ser amigo pessoal, mas no relacionamento profissional tem que dar atenção (Fisioterapeuta 01).

Eu sei o que é dor e o que é sofrimento, eu procuro receber o paciente da melhor maneira possível, criando um vínculo com ele sem intimidades, é claro, para que essa uma hora que ele passa aqui, ele se sinta bem, que tire suas dúvidas e que confie no profissional, que até brinque às vezes, tendo um respeito, mas também com liberdade (Fisioterapeuta 02).

A redução fenomenológica indicou três focos problemáticos para o fenômeno da adesão ao tratamento na fisioterapia. 0 primeiro foco problemático é a necessidade do cuidado integral, que compreende a forma de abordagem fisioterapêutica durante o tratamento, considerando o paciente em todas as suas dimensões (físicas, psíquicas e sociais). 0 segundo foco problemático é a dificuldade na comunicação paciente-terapeuta, que abrange os aspectos da relação que se estabelece entre terapeutas e pacientes. 0 terceiro foco problemático são as contingências socioeconômicas que perpassam as limitações financeiras dos pacientes atendidos pelo SUS e constituem fatores ligados à permanência ou ao abandono da fisioterapia. Esses três focos problemáticos serão discutidos a seguir, à luz de achados empíricos e propostas teóricas já estabelecidos na literatura científica.

\section{Discussão}

A interpretação fenomenológica ampliou os focos problemáticos apresentados na redução, propondo uma compreensão teórica do fenômeno da relação fisioterapeuta-paciente e seu impacto na adesão ao tratamento na fisioterapia, com base em três aspectos: o cuidado integral, a comunicação e os fatores socioeconômicos de cada paciente.

A adesão não se limita ao conhecimento e reconhecimento da doença músculoesquelética e à escolha do procedimento mais adequado à reabilitação. A adesão na fisioterapia envolve questões ligadas ao cuidado integral, cercado de uma abordagem terapêutica e avaliação multifatorial; ao desenvolvimento e aperfeiçoamento continuado da comunicação e da relação que se estabelece em todos os níveis interpessoais do tratamento; e à consideração dos fatores socioeconômicos de cada paciente.
Portanto, aderir ao tratamento na fisioterapia parece um fenômeno primordialmente qualitativo, ou seja, um processo que vai além da realização das 10 ou 20 sessões prescritas pela equipe. Envolve mais do que uma disfunção músculoesquelética a ser resolvida. A adesão compreende uma situação em que um indivíduo busca o serviço do centro de reabilitação, carregando consigo uma série de sentimentos, perspectivas, emoções, dores e angústias, que podem, na maioria dos casos, se aproximar ou estar relacionadas de alguma forma ao adoecimento de ordem física.

0 cuidado integral perpassa questões que envolvem a tomada de decisões tanto por parte dos terapeutas quanto dos pacientes. Cuidar integralmente de um indivíduo significa percebê-lo como um ser social, físico e emocional, que carrega consigo todos os medos, angústias e frustrações por estar doente naquele momento. 0 paciente é um indivíduo que apresenta uma história e uma personalidade únicas. Sua carga genética, assim como as suas experiências de vida, terá influência sobre a sua personalidade e sobre a forma como reage à doença. Está no próprio indivíduo o motivo de sua doença $(19,20)$.

No presente estudo, os dados sugerem que o tratamento recebido pelos pacientes, apesar de ter sido considerado satisfatório, foi alvo de uma série de pontuações e críticas, dentre elas a reivindicação por mais tempo de contato com os fisioterapeutas e a necessidade de serem ouvidos em maior profundidade quanto aos aspectos emocionais que podem estar envolvidos com os acometimentos de ordem física. A expectativa por mais toque, mais atenção e maior disponibilidade para serem ouvidos expressa uma necessidade de tratamento mais abrangente, tirando o foco apenas da parte física lesionada. Os fisioterapeutas revelaram, por seu lado, estar conscientes da necessidade desse tipo de abordagem sistêmica.

No entanto, percebe-se um desencontro entre a fala e a prática diária, visto que não há tempo suficiente para a realização de uma anamnese aprofundada com cada paciente, nem a chance de ouvi-lo um pouco mais a cada sessão. Esse desajuste surge a partir do momento em que um mesmo fisioterapeuta precisa atender cerca de cinco pacientes por hora de terapia, o que de fato o impossibilita de oferecer um tratamento de maneira efetivamente integral. Soma-se a isso a automatização do atendimento fisioterapêutico, pois nem mesmo os profissionais se dão conta da perda de qualidade em atendimento, considerando muitas vezes que, apesar do pouco tempo com o paciente $\mathrm{e}$ 
as altas demandas, a reabilitação é satisfatória para quem oferece e para quem recebe o cuidado fisioterapêutico. Esse dado corrobora estudos anteriores que consideram o ritmo intenso de trabalho e a necessidade de atender um número máximo de pacientes, como contribuintes à perpetuação do modelo biomédico de atendimento e de uma visão quantitativa do processo de adesão $(2,20-22)$.

Em se tratando do modelo biomédico, pouco se diz sobre o sofrimento e sobre como o indivíduo se percebe diante do adoecimento, já que seus procedimentos e sua forma de abordagem terapêutica acabam por resumir as pessoas, as coisas, a vida e os acontecimentos do mundo às medidas de seus padrões de referência, em que o mais importante é a parte doente e não o todo (23). No entanto, quando se parte do pressuposto de que todas as doenças são psicossomáticas, já que corpo e mente são inseparáveis anatômica e funcionalmente $(12,13,24)$, constitui-se um erro tentar dissociar os problemas psicológicos e emocionais dos transtornos orgânicos. 0 paciente expressa, por meio de palavras, gestos, posturas e ações, além de características pessoais, a forma como a disfunção se manifesta.

Desse modo, identificar se um paciente apresenta fatores emocionais ligados ao processo de adoecimento, como causa ou como consequência, requer que a formação do fisioterapeuta necessariamente seja voltada também para o entendimento e avaliação do impacto dos aspectos psicossociais do indivíduo e, especialmente, para os aspectos comunicacionais do relacionamento fisioterapeuta-paciente. Estudos já publicados em diversas áreas, como na psicoterapia (25), na enfermagem (26-27), na medicina (18$28)$ e na fisioterapia (10-12), sugerem que a efetividade de um tratamento, assim como a adesão, está estreitamente relacionada à comunicação e, principalmente, à qualidade da relação entre terapeuta e paciente. Portanto, o cuidado integral de um paciente requer, além das habilidades técnico-científicas, a capacidade de estabelecer comunicação adequada entre todos os envolvidos no processo de reabilitação, desde o momento do acolhimento do paciente, passando pela triagem, durante as sessões, até o dia da alta fisioterapêutica. Tanto aqueles que recebem o tratamento como aqueles que tratam, veem na comunicação uma ferramenta essencial ao sucesso da reabilitação. A relação de qualidade entre terapeuta e paciente contribui para o conhecimento mais aprofundado daquele que é tratado, além da expansão do conhecimento de quem trata, sobre a melhor forma de reabilitar o paciente e de avaliar o tipo de abordagem terapêutica que é oferecida a cada um (29-32).

0 paciente é alguém que se comunica e necessita de atenção especial não só como portador de uma patologia (33). Segundo Leon (34), para que o profissional da área da saúde seja bom é necessário priorizar a comunicação com o paciente. A relação criada pelo profissional com seu paciente, quando afetuosa e valorizada, favorece o desenvolvimento de estados emotivos positivos, que facilitam a reabilitação. Quanto mais o fisioterapeuta entende o paciente, maior é a possibilidade de ajudá-lo, tratá-lo e incentivá-lo a realizar todo o tratamento. Quanto maior é a compreensão das necessidades, das capacidades e desejos do paciente pelos profissionais da área da saúde, maior será o sucesso obtido no seu tratamento $(2,20,21,23)$. Contudo, isso não significa que os fisioterapeutas e demais profissionais tenham de mudar sua prática, exercendo a função de um psicólogo, mas devem estar mais atentos à comunicação com o paciente e compreender em que medida podem usar o diálogo a favor do tratamento e quando devem convidar o psicólogo a participar do processo de reabilitação. 0 fato de o relacionamento ser considerado um aspecto mais relevante do que a habilidade técnica do fisioterapeuta durante a reabilitação também não deve ser entendido como uma afirmação de que o tratamento sem resultados terapêuticos seja considerado bom. A preponderância da qualidade do relacionamento sobre a habilidade técnica pode ser explicada pelo fato de que, até que haja a remissão dos sintomas e a devolução das funções perdidas, a relação satisfatória que se estabelece entre fisioterapeuta e paciente é o grande facilitador da adesão ao tratamento e, por consequência, um fator essencial para o sucesso da reabilitação.

A dificuldade tanto por parte de fisioterapeutas quanto por parte dos pacientes em descrever e classificar o tipo de relacionamento que se estabelece entre eles parece indicar uma dificuldade dos primeiros em lidar com a informação psicológica, especialmente relacionada a estados emocionais (angústias, medos, frustrações, carências, entre outros), trazida pelos últimos à terapia. Essa dificuldade perpetua o distanciamento entre quem trata e quem recebe a atenção, e reforça o receio dos fisioterapeutas em estabelecer vínculos afetivos que possam se tornar uma relação de dependência pelo tratamento e ou pela relação que o paciente estabelece com o profissional. Esse ponto 
de equilíbrio entre o vínculo saudável e o vínculo dependente é visto como um desafio aos profissionais de saúde de diversas áreas (10). No caso da fisioterapia, parece fundamental compreender as características da relação que se estabelece entre quem trata e quem é tratado. 0 entendimento dessas questões deve iniciar-se no processo de formação profissional, sendo sua prática estimulada em todo o exercício da profissão. Sugere-se, com base em nossos achados, a busca por mais pesquisas que discutam os aspectos psicológicos presentes na adesão à fisioterapia. Os resultados dessas pesquisas precisam deixar o mundo estritamente acadêmico e alcançar as equipes, os profissionais e os pacientes estudados. Que esses caminhos construam-se no sentido de perceber em profundidade em que medida o profissional possa usar a relação interpessoal como fator favorável ao processo terapêutico.

\section{Considerações finais}

A presente pesquisa tem caráter qualitativo e, portanto, diferentemente da pesquisa quantitativa, que permite precisão e generalização em atribuição, ou, em outros termos, a predição de resultados, a pesquisa qualitativa possibilita exatidão e abstração na descrição, isto é, uma adequada representação do fenômeno investigado $(17,35-37)$.

0 estudo pôde compreender, com base na percepção de pacientes e de fisioterapeutas, quais os aspectos envolvidos no relacionamento entre aquele que trata e aquele que é tratado, indicando que a qualidade da relação que se estabelece entre ambos é fundamental ao processo de adesão à fisioterapia. Um processo que deve ser fundamentado não apenas no número de sessões, mas na qualidade de cada uma delas.

Considerando a diversidade de atuações no campo da fisioterapia, novos estudos devem ser realizados com pacientes e fisioterapeutas de diferentes instituições, a fim de compreender os fatores que contribuem para o processo de adesão e a relevância do relacionamento fisioterapeuta-paciente para o sucesso da reabilitação.

\section{Agradecimentos}

À Fundação de Amparo à Pesquisa do Espírito Santo (Fapes), pela bolsa de estudos concedida.

\section{Referências}

1. Garcia A. (Org.). Relacionamento interpessoal e saúde: avanços recentes nos estudos sobre relacionamento médico-paciente. In: Garcia A. (Org.). Relacionamento interpessoal: olhares diversos. Vitória: GM; 2005. p. 41-9.

2. Caprara A, Rodrigues J. A relação assimétrica médicopaciente: repensando o vínculo terapêutico. Ciência e Saúde Coletiva. 2004;9(1):139-46.

3. Oliveira VZ, Gomes WB. Comunicação médico-paciente e adesão ao tratamento em adolescentes portadores de doenças crônicas. Estudos de Psicologia. 2004; 9(3):459-69.

4. Cruz EMTN. Formando médicos da pessoa: o resgate das relações médico-paciente e professor-aluno. Rev Bras Educ Med. 1997;21(2/3):22-8.

5. Wulff HR, Pedersen SA, Rosemberg R. La filosofia della medicina. Milano: Raffaelo Cortina; 1995.

6. Marinho PEM. Escutar: aspecto relevante da relação fisioterapeuta-paciente na fisioterapia. Fisioter Mov. 1995;7(2):59-62.

7. Loyola MA. Médicos e curandeiros: conflito social e saúde. São Paulo: Difel; 1984.

8. Ferreira J. O corpo sígnico: representações sociais sobre corpo, sintomas e sinais em uma vila de classes populares [dissertação]. Porto Alegre: Universidade Federal do Rio Grande do Sul; 1993.

9. Ribeiro J, Moraes MVM, Beltrame TS. Tipo de atividade e relação interpessoal estabelecida entre fisioterapeuta e criança com paralisia cerebral no contexto de intervenção fisioterapêutica. Dynamis. 2008;14(1):89-95.

10. Sanguin FPS, Vizzoto MM. As variáveis psicológicas no processo de adesão ao tratamento fisioterapêutico. Mudanças, Psicologia da Saúde. 2007;15(1):13-22.

11. Moreira V, Nogueira FNN, Rocha MAS. Leitura fenomenológica do adoecer em pacientes do serviço de fisioterapia do núcleo de atenção médica integrada, Universidade de Fortaleza. Estudos de Psicologia. 2007; 24(2):191-203.

12. Marinho AP, Fiorelli JO. Psicologia na fisioterapia. São Paulo: Atheneu; 2007.

13. Marinho PEM. Refletindo sobre a expressividade da dor e a relação terapeuta-paciente. Fisioter Mov. 2005; 18(2):73-9. 
14. Subtil MML, Souza ML. Adesão ao tratamento fisioterapêutico: uma análise fenomenológico-semiótica da percepção de pacientes e terapeutas [dissertação]. Espírito Santo: Universidade Federal do Espírito Santo; 2010.

15. Cresweel JW. Qualitative inquiry e research design: choosing among five aprroaches. London: Sage; 2007.

16. Gil AC. Como elaborar projetos de pesquisa. São Paulo: Atlas; 2002.

17. Gomes WB. (Org.). Fenomenologia e pesquisa em psicologia. Porto Alegre: Ed. da UFRGS; 1998.

18. Lanigan R. The human science of communicology. Pittsburgh: Duquesne University Press; 1992.

19. Perestrello D. A medicina da pessoa. Rio de Janeiro: Atheneu; 1996.

20. Tahka W. O relacionamento médico-paciente. Porto Alegre: Artes Médicas; 1988.

21. Olivieri DP. O ser doente: dimensão humana na formação do profissional de saúde. São Paulo: Moraes; 1985.

22. Moreira TMM, Araújo TL. Sistema interpessoal de Imogenes King: as relações entre pacientes com hipertensão não aderentes ao tratamento e profissionais de saúde. Acta Paul Enf. 2002;15(3):35-43.

23. Fishman T. The 90-second intervention: a patient compliance mediated technique to improve and control Hypertension. Public Health Reports. 1995;110(2): 173-8.

24. Castiel LD. O buraco e o avestruz: a singularidade do adoecer humano. Campinas: Papirus; 1994.

25. Mello SL. Psicologia e profissão em São Paulo. São Paulo: Ática; 1983.

26. Jardim AP, Souza ML, Gomes WB. O self dialógico e a psicoterapia: uma compreensão dialógica da relação terapeuta-paciente. Contextos Clínicos. 2009; 2(1):1-10.

27. Esperidião MA, Trad LAB. Avaliação de satisfação de usuários: considerações teórico-conceituais. Cad Sáude Pública. 2006;22(6):1267-76.
28. Hoga LAK. A dimensão subjetiva do profissional na humanização da assistência à saúde: uma reflexão. Rev Esc Enferm USP. 2004;3(1):13-20.

29. Lemme AC. Ouvindo e encantando o paciente. Rio de Janeiro: Qualitymark; 2005.

30. Balint M. O médico, seu paciente e a doença. São Paulo: Atheneu; 2007.

31. Abdo CHN. Armadilhas da comunicação: o médico, o paciente e o diálogo. São Paulo: Lemos; 1996.

32. Silva LK, Sena RR. Integralidade do cuidado na saúde: indicações a partir da formação do enfermeiro. Rev Esc Enferm USP. 2008;42(1):48-56.

33. Stone D, Patton B, Heen S. Conversas difíceis. Rio de Janeiro: Elsevier; 2004.

34. Costa RO Jr, Sandoval JMH. O cuidado a saúde e a comunicação: um olhar da perspectiva de estudantes de fisioterapia. Anais do 25ํㅡㄴ Intercom, Congresso de Ciências da Comunicação. Salvador: Intercom; 2002.

35. Leon A. Ética em medicina. Barcelona: CientíficoMedicina; 1973.

36. Souza ML, Gomes WB, McCarthy S. Reversible relationship between quantitative and qualitative data in selfconsciousness research: a normative semiotic model for the phenomenological dialogue between data and capta. Quality and Quantity. 2005;39:199-215.

37. Souza ML, Gomes WB. Evidência e interpretação em pesquisa: as relações entre quantidades e qualidades. Psicologia em Estudo. 2003;8(2):83-92.

Recebido: 19/10/2010

Received: 10/19/2010

Aprovado: 23/05/2011

Approved: 05/23/2011 